\title{
Designing a Socially Assistive Companion Robotic Wheel Chair: RoboChair
}

\author{
Chandimal Jayawardena ${ }^{1}$, Nilufar Baghaei ${ }^{1}$, Kathiravelu Ganeshan ${ }^{1}$, and Abdolhossein Sarrafzadeh ${ }^{1}$
}

\begin{abstract}
Developing socially assistive robots is an emerging interdisciplinary research area, which requires collaboration between a wide range of disciplines. Among recent research projects, there have been attempts to develop assistive robotic solutions to solve various health and social issues. In most current research attempts to design socially assistive robots, the focus is on designing new robotic agents that can interact with people by various means.
\end{abstract}

Since people do not have much experience with robots, usually extensive field trials are conducted in order to assess the usability of these robots. However, determining the usability of these robotic agents is a difficult task, since the results of field trials are not always conclusive. In this paper, an attempt to overcome the difficulty of evaluating usability of assistive robots is presented.

This paper presents the design of the first version of a companion robot called RoboChair. RoboChair is in the form of a wheel chair. But, functionally it is a socially assistive companion robot.

The proposed robotic chair is a mobile robot that can carry a person. It is equipped with several measuring devices for taking clinical measurements mentioned above. In addition to that, it is equipped with several sensors for obstacle avoidance, map building, localization, detecting humans etc. It is also equipped with motor controllers and other actuators for motion control.

The robot chair is capable of engaging users with interactive dialogs through a touch screen and by using human-robot interaction techniques. It has a scalable modular architecture so that adding new hardware and software modules is straightforward. The software framework is based on Robot Operating System (ROS) open source robotic middleware.

RoboChair is controlled by a distributed controller that spans multiple hardware devices and multiple operating systems.

\section{INTRODUCTION}

Developing socially assistive robots is an emerging interdisciplinary research area, which requires collaboration between a wide range of disciplines, including robotics, health sciences, psychology, gerontology, and human-computer interaction [1] [2]. In recent years, there is an increased interest in this area due to multiple reasons. Increasing capabilities of mobile service robots, increasing needs of people for assistance, and increasing numbers of older population around the world are some of the important reasons [3]. On the other hand, there is an increasing shortfall in numbers of health professionals and caregivers [4].

Among recent research projects, there have been attempts to develop assistive robotic solutions to solve various health and social issues. Mobility aids [5], manipulation aids [6], therapeutic aids [7], surgical robots [8], physical and mental

\footnotetext{
${ }^{1}$ C. Jayawardena, N. Baghaei, K. Ganeshan, and A. Sarrafzadeh are with the Department of Computing, Unitec Institute of Technology, New Zealand cjayawardena at unitec.ac.nz
}

rehabilitation robots [9] [10], medication reminding robots [11] and elder-care robots [12] are some examples . Among these solutions, 'socially assistive robots' belong to a distinct category.

Socially assistive robots are different from social robots and entertainment robots, which provide relatively simple human-robot interactions. In contrast, socially assitive robots are expected to provide a broad range of services to support daily activities of users.

In most current research attempts to design socially assistive robots, the focus is on designing new robotic agents that can interact with people by various means [13], [14], [15]. Since people do not have much experience with robots, usually extensive field trials are conducted in order to assess the usability of these robots. However, determining the usability of these robotic agents is a difficult task, since the results of field trials are not always conclusive [14], [16]. The difficulty of assessing usability directly affects product development and commercialization.

\section{A. An alternative approach for developing useful socially assistive robots}

In this paper, an attempt to overcome the difficulty of evaluating usability of assistive robots is presented. The authors suggest a novel approach to develop socially assistive robots and the current status of a research in that direction is presented.

The authors approach is to convert an already useful assistive device to a socially assistive robot by embedding various robotic features. The objective of the research presented on this paper is to convert a normal powered wheel chair to a socially assistive companion robot. Functionally, this wheel chair will act as a socially assistive companion robot, while fulfilling its function as a wheel chair.

\section{SySTEM OVERVIEW}

This paper presents the design of the first version of a companion robot called RoboChair. RoboChair is a project conducted at the Unitec Institute of Technology, New Zealand.

RoboChair project is inspired mainly by the HealthBots project of the University of Auckland [14]. The motivation for designing this robotic chair comes from the service robot domain, in particular from health care robot domain. Currently, there many robots are being developed for health monitoring and assessment. HealthBot robot can do medication reminding, vital signs measurement, falls detection among many other things [16]. 
RoboChair is in the form of a wheel chair. But, functionally it is a socially assistive companion robot. The features included in the first version are as follows:

- Voice controlled navigation using fuzzy voice commands

- Vital signs (blood pressure, blood oxygen saturation, body weight, and bio impedance

- Cardiovascular risk prediction

- Communicating with friends, family, and health professionals

- Provide companionship through multi-modal interactions

These features are very similar to the features commonly associated with personal robots. However, the design philosophy of RoboChair is quite different. The authors argue that it is more beneficial to embed features of socially assistive companion robots into already useful devices rather than building completely new robots. Since the core device is already in use, it is expected that the resistance to user acceptance would be minimal. However, this hypothesis should be tested through user trials, which will be the next step of this research.

The following key requirements were considered in the design of RoboChair:

- Low cost: Since this robot is intended to be a personal robot, cost is a primary consideration. Therefore, simpler and cheaper technologies were used.

- Simple user interface: Since the target user group was older or disabled people simple user interfaces were used.

- Simple operation: All services provided by the robot could be accessed via a touch screen. Speech synthesis was used, but speech recognition was not used.

- Voice controlled navigation: For the convenience of the users with physical limitations, voice controlled navigation was included.

- Robustness: The working environment of the service robot is a domestic setting, where technical support is not readily available. Therefore, special attention was paid to the robustness.

- Open-source software: For software development, opensource software was used. In particular, Robot Operating System (ROS) was used as the middleware.

Another important design consideration of RoboChair is the ability to measure body weight. This is a limitation of almost all existing socially assistive robots designed for the health care domain. Most healthcare robots help users in various areas related to health. Medication management, help quit smoking are some examples. However, weight is an important measurement in health and inability to measure the weight of a user is a major limitation.

The first version of RoboChair is shown in Fig. 2(a).

\section{ROBOT HARDWARE}

The robot presented in this paper is a result of an ongoing project at the Unitec Institute of Technology, New
Zealand. The robot hardware consists of a differential drive mobile platform, a note book computer (main controller), a tablet computer (GUI), sonar sensors, microphone, speakers, a kinect sensor, a laser range finder, a load cell and vital signs measuring devices. RoboChair is powered by a $24 \mathrm{v}$ Li-Polymer battery.

The tablet mounted on a rotatable mount is the main mode of interaction with the users. It displays instructional text, graphics, and video as well as takes user inputs by means of touch events,

The kinect sensor is used for people detection using cobpeople-detection ROS package [17]. The robots synthetic speech was generated through Festival open-source speech synthesis system [18].

\section{SOFTWARE ARCHITECTURE}

The software architecture is based on the ROS open-source framework. An overview of the architecture is shown in Fig. 2(b).

The architecture comprised of a main controller, GUI generator, several distributed components and robot hardware.

\section{A. Main controller}

The main controller shown in Fig. 2(b) controls the core behaviour of the robot. It is a Finite State Machine (FSM) created using SMACH package of ROS [17]. The main control thread of the robot is in the FSM and it controls and coordinates all the other distributed components.

Each state is associated with actions, GUI, and events. This is illustrated in Fig. 1. This representation has similarities

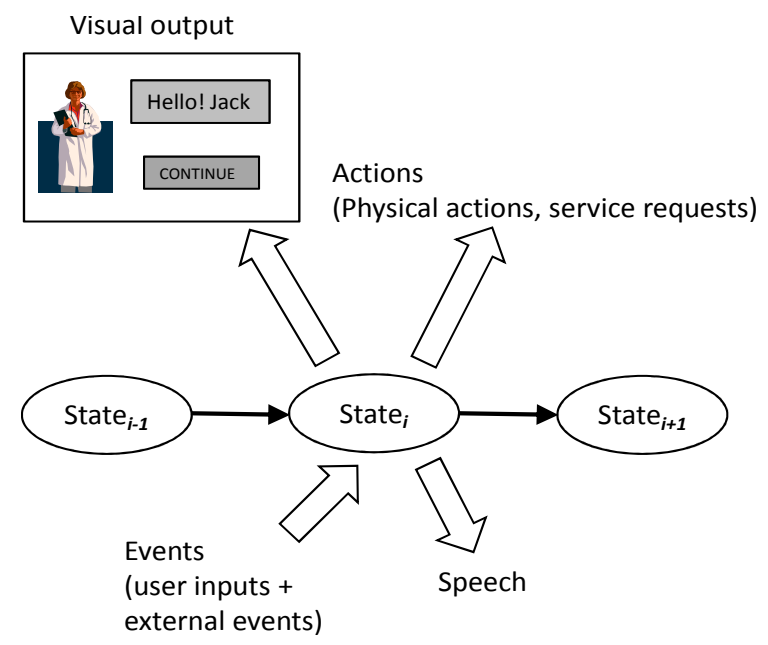

Fig. 1. State behavior

with the finite-state-machine based behaviour presented in [16]. However, there are major difference and improvements in RoboChair software architecture, when compared to HealthBots Version 2 architecture presented in [16]

In HealthBots Version 2 architecture, robot behaviour and screen layout definitions are in a single FSM and behaviour generation and screen generation are done by a single monolithic software. In RoboChair architecture, the robot 
behaviour description and screen layout generation are separated. Still both robot behaviour and screen layout generation are controlled by a single FSM due to reasons described in [16], but the actual screen generation and all front-end activities are done by a separate software component (GUI generator), coordinating with the main FSM.

It was decided to combine the definitions of robot actions and screen generation due to following reasons.

Usually, in robotic applications, the GUI is not included in the robot behaviour design. Instead, the focus is on robot behaviour such as path planning, navigation, and other actions. However, in service robot applications, the GUI is a dominant part of the robot behavior, since the user experience highly depends on the audio visual output and the interaction with the robot. Therefore, to have effective human-robot interaction, robot actions, user inputs, as well as changes in GUI, should be synchronized [16].

\section{B. GUI generator}

GUI generator is a ROS component implemented using QT-ROS package [17]. IT uses the widely used QT framework for GUI generation.

In RobotChair GUI there are no fixed components. The main controller determines what should be displayed on the GUI depending on the current state of the main controller and sends a message to GUI generator. This is an XML formatted message that completely describes the screen layout and components. Upon receiving this, the GUI generator dynamically generates the screen layout required by the current state of the robot.

\section{Distributed components}

There are several distributed ROS components to implement various robot functions. Following are the components in the current implementation:

- Navigation: This components receives fuzzy voice controlled commands described below and converts these commands into motor control commands. Then it sends messages to the micro controller responsible for sending PWM signals to motor drives.

- Weight measurement: This components receives commands for acquiring weight. IT communicates with the micro controller responsible for reading the load-cell output. It returns the weight as a ROS message.

- Blood pressure and pulse rate measurement: This components received commands for acquiring blood pressure of a user and then send control signals to a blood pressure meter. Blood pressure measurement consists of several steps and this component is responsible for monitoring and controlling all steps and finally taking the blood pressure measurement. It returns the systolic blood pressure, diastolic blood pressure, and pulse rate as a single ROS message.

- Blood oxygen saturation measurement: Similar to blood pressure measurement, this module controls a blood oxygen meter and returns the reading.
- People detection: This component detects the number of people in front of the robot as a ROS message. This information is used by the main controller to generate engaging dialogs.

- Text-to-speech: This components receives text to be spoken and then generates voice using Festival.

\section{Fuzzy voice controlled navigation}

For voice controlled navigation, a fuzzy coach-player approach is used [19], [20], [21], [22], [23] is used. In this approach, in a voice command there are two parts; action and action modification. For the whee chair, go forward, go backward, turn right, and turn left are used as actions and very slow, slow, fast, and very fast are used as action modifications. Since the main focus of this paper is the overall design of the robot, details of voice controlled navigation are not presented here.

\section{EXPERIMENTS AND CONCLUSION}

The first version of the RoboChair is currently being tested in the laboratory. This robot can be used in multiple scenarios. Following are some examples:

- In a waiting room of a clinic: Typically, patients have to wait in the waiting room of a clinic for some time. RoboChair can be placed in such a place to attract people to use it. By doing so, it can measure vital signs without any help from a nurse and upload results to the information system, thus saving time. On the other hand, it can provide some health assessments like cardiovascular risk prediction and encourage users to reduce their body weight.

- As a personal wheel chair: Since the RoboChair has all the features of a normal power chair, it can be used as a personal device. Since it has all the other additional robotic features, it will be more useful to its users than a normal power chair.

- In a rest home: RoboChair can be used in a rest home as well. It can be a good companion to lonely people since it has common features of a companion robot. Authors argue that RoboChair would be more useful and attractive to people than a normal powered chair.

Although RoboChair can be used in these difference scenarios, the first scenario above was selected to perform the first experiments. The experimental scenario is depicted in Fig. 3.

Initially, the robot waits in an idle state (the location is assume to be a waiting room of a clinic). In the idle state the robot displays a welcome message on the screen. If a potential user approaches the robot, it is detected through the sensors. Then the robot tries to engage the user. For example, the robot says "Hello, how are you today?." Then a sequence of interactions follow and the RoboChair invites the user to sit on the chair and use the services provided through the menu. In the current prototype, blood pressure measurement, blood oxygen saturation measurement, body weight measurement, entertainment, and voice controlled navigation are present. In this scenario (in a waiting room of a clinic), 


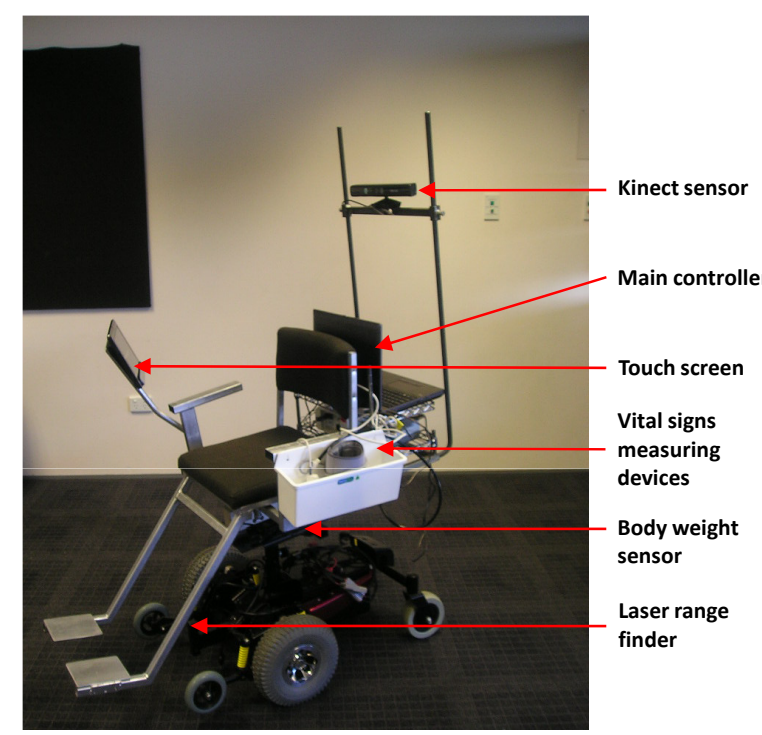

(a) RoboChair

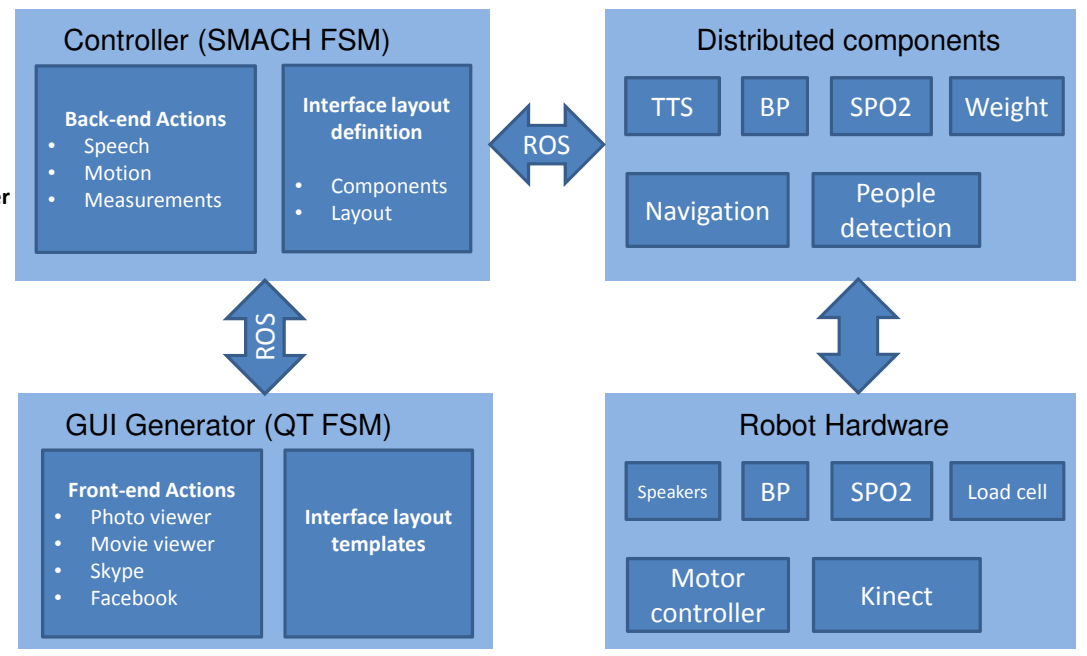

(b) Software architecture

Fig. 2. RoboChair and software architecture

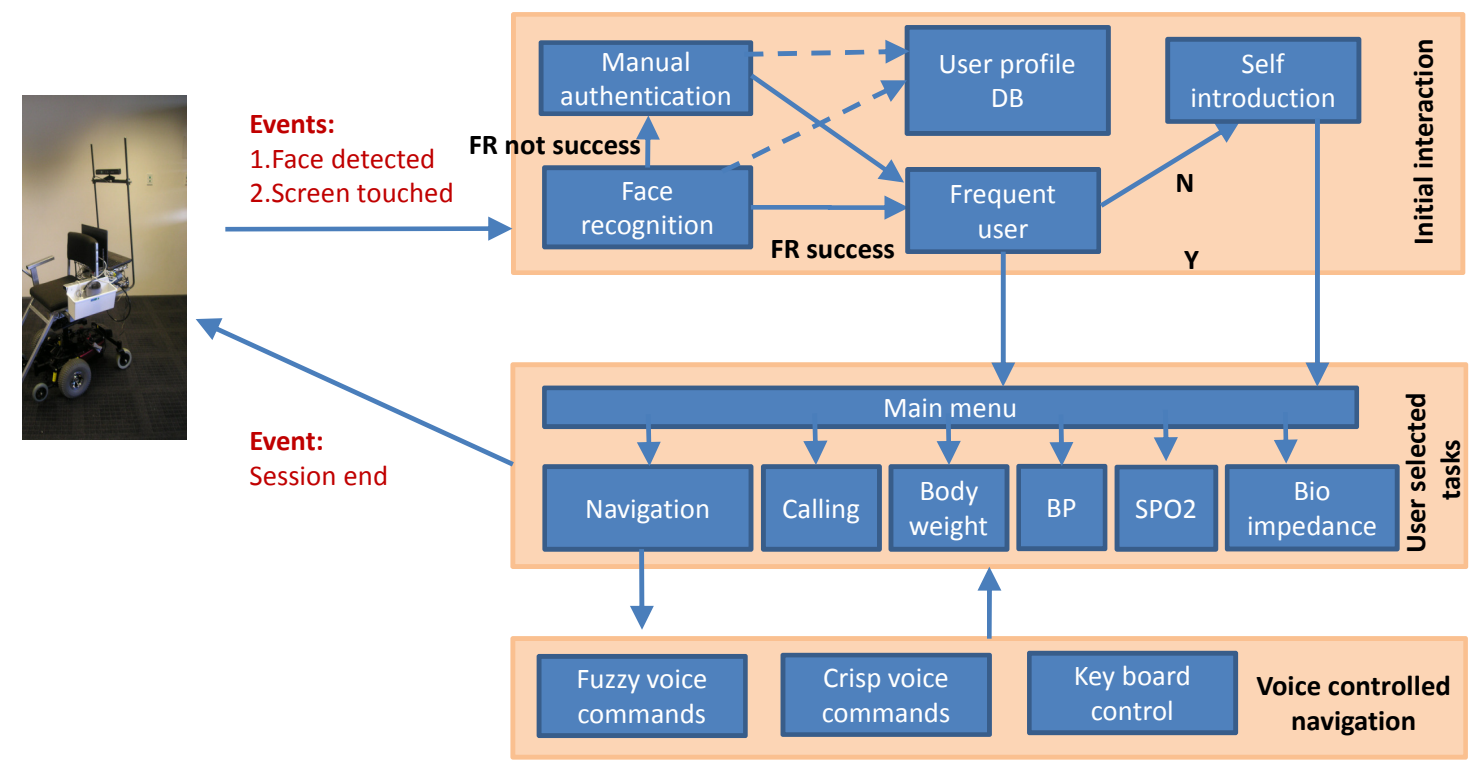

Fig. 3. Experimental scenario

blood pressure, blood oxygen saturation, pulse rate, and body weight measurements are the most relevant functions. Once these measurements are taken, then measured values can be uploaded to the information system of the clinic.

A typical user interaction session is shown in Fig. V.

From these experiments, from user feedback and from authors' experience, several future improvements were identified. The key ones are given below:
- Integration with social networks: Being a companion robot, RoboChair can help users with disabilities to be engaged with the society through social networks. Although the users may not be able to use social networks directly due to physical limitations, RoboChair can help them to connect to social networks and use their features.

- Video conferencing: This is an essential feature, specially for lonely people in rest homes. The video confer- 


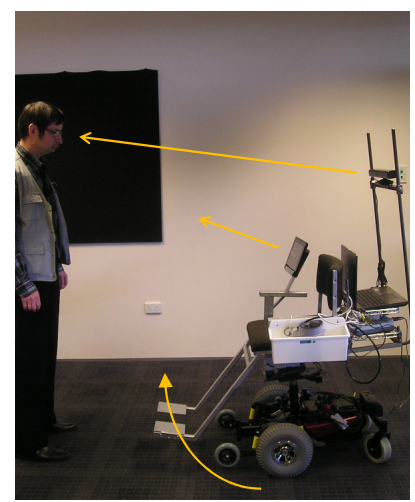

(a) Initial interaction 1 .

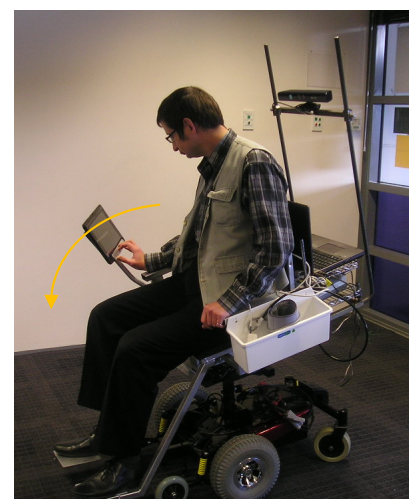

(c) Initial interaction 1 .

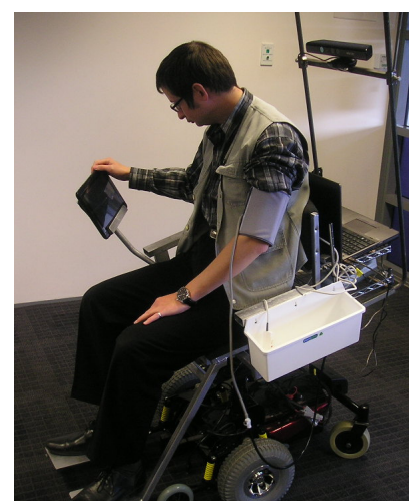

(e) Initial interaction 1 .

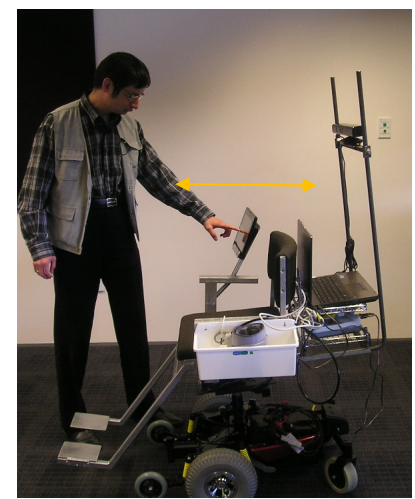

(b) Initial interaction 1 .

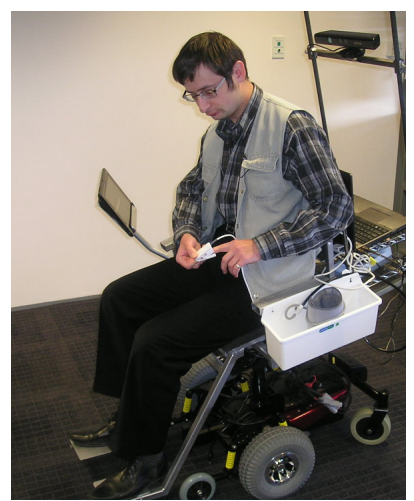

(d) Initial interaction 1.

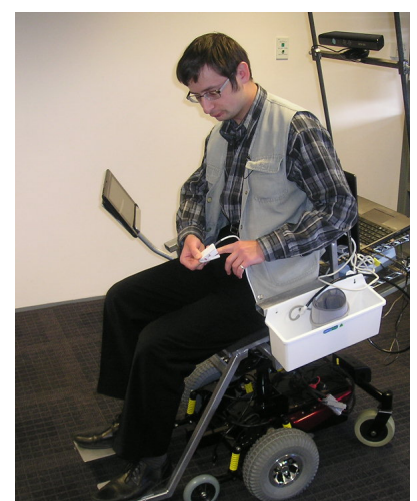

(f) Initial interaction 1.
Fig. 4. HealthBot robot and test scenarios.

encing feature can be used to communicate with family members, friends and with health care professionals.

- Health monitoring and risk prediction: This is one of the key application areas of RoboChair, once completed. By measuring some vital clinical parameters, it is possible to predict things such cardiovascular risk. Being a companion a robot, RoboChair can be used to provide risk information to users, educate them, and help them to reduce the risk through education.
- Health educational content: As a companion robot, providing educational information such as medication information will enhance the usefulness of the robot. [11]

- Integration with sensor networks (intelligent environment): By integrating with sensor networks, it is possible to extend the usefulness of RoboChair by providing services such as falls monitoring [14].

- HL7 compliance: HL7 is an application layer protocol used by health information systems. HL7 compliance is an essential feature of RoboChair since it is necessary to communicate with existing health information systems.

The above features have been given priority in the current development work of RoboChair.

\section{REFERENCES}

[1] L. Boccanfuso and J. M. O'Kane, "Charlie : An adaptive robot design with hand and face tracking for use in autism therapy," International Journal of Social Robotics, vol. 3, pp. 337-347, 2011.

[2] Y. Yamaji, T. Miyake, Y. Yoshiike, P. R. S. Silva, and M. Okada, "Stb: Child-dependent sociable trash box," International Journal of Social Robotics, vol. 3, pp. 359-370, 2011.

[3] W. Lutz, W. Sanderson, and S. Scherbov, "The coming acceleration of global population ageing," Nature, vol. 451, no. 7179, pp. 716-719, 2008, 10.1038/nature06516.

[4] Establishing and Monitoring Benchmarks for Human Resources for Health: the Workforce Density Approach, ser. Spotlight on Statistics, no. 6, World Health Organization, Department of Human Resources for Health, WHO, 2008.

[5] Y. Hasegawa, J. Jang, and Y. Sankai, "Cooperative walk control of paraplegia patient and assistive system," in Proc. IEEE/RSJ International Conference on Intelligent Robots and Systems, Oct. 2009, pp. 4481-4486.

[6] J. Kofinan, X. Wu, T. Luu, and S. Verma, "Teleoperation of a robot manipulator using a vision-based human-robot interface," IEEE Transactions on Industrial Electronics, vol. 52, no. 5, pp. 1206-1219, Oct. 2005.

[7] K. Kiguchi, T. Tanaka, and T. Fukuda, "Neuro-fuzzy control of a robotic exoskeleton with emg signals," IEEE Transactions on Fuzzy Systems, vol. 12, no. 4, pp. 481-490, Aug. 2004.

[8] W. Shin and D. Kwon, "Surgical robot system for single-port surgery with novel joint mechanism," IEEE Transactions on Biomedical Engineering, vol. - , no. - , pp. -

[9] H. Krebs, B. Volpe, D. Williams, J. Celestino, S. Charles, D. Lynch, and N. Hogan, "Robot-aided neurorehabilitation: A robot for wrist rehabilitation," IEEE Transactions on Neural Systems and Rehabilitation Engineering, vol. 15, no. 3, pp. 327-335, 2007.

[10] A. Di Nuovo, D. Marocco, A. Cangelosi, V. De La Cruz, and S. Di Nuovo, "Mental practice and verbal instructions execution: A cognitive robotics study," in Proc. The 2012 International Joint Conference on Neural Networks (IJCNN), 2012, pp. 1-6.

[11] P. Tiwari, J. Warren, K. Day, B. MacDonald, C. Jayawardena, I. H. Kuo, A. Igic, and D. C., "Feasibility study of a robotic medication assistant for the elderly," in Proc. Australasian Conf. User Interface (AUIC), 2011, pp. - .

[12] I. H. Kuo, C. Jayawardena, E. Broadbent, R. Q. Stafford, and B. A. MacDonald, "Hri evaluation of a healthcare service robot," in Social Robotics, ser. Lecture Notes in Computer Science, S. Ge, O. Khatib, J.-J. Cabibihan, R. Simmons, and M.-A. Williams, Eds. Springer Berlin Heidelberg, 2012, vol. 7621, pp. 178-187.

[13] A. Tapus, M. Mataric, and B. Scasselati, "Socially assistive robotics [grand challenges of robotics]," IEEE Robotics Automation Magazine, vol. 14, no. 1, pp. 35-42, 2007.

[14] C. Jayawardena, I. H. Kuo, U. Unger, A. Igic, R. Wong, C. Watson, R. Stafford, E. Broadbent, P. Tiwari, J. Warren, J. Sohn, and B. MacDonald, "Deployment of a service robot to help older people," in Proc. IEEE/RSJ International Conference on Intelligent Robots and Systems (IROS), Oct. 2010, pp. 5990-5995. 
[15] R. Stafford, E. Broadbent, C. Jayawardena, U. Unger, I. H. Kuo, A. Igic, R. Wong, N. Kerse, C. Watson, and B. MacDonald, "Improved robot attitudes and emotions at a retirement home after meeting a robot," in Proc. IEEE RO-MAN, 2010, pp. 82-87.

[16] C. Jayawardena, I. Kuo, C. Datta, R. Stafford, E. Broadbent, and B. MacDonald, "Design, implementation and field tests of a socially assistive robot for the elderly: Healthbot version 2," in Proc. 4th IEEE RAS EMBS International Conference on Biomedical Robotics and Biomechatronics (BioRob), 2012, pp. 1837-1842.

[17] (2013, Jul.) Robot operating system (ros). [Online]. Available: http://www.ros.org

[18] A. W. Black, P. Taylor, and R. Caley. (2013, Jul.) The festival speech synthesis system. [Online]. Available: http://www.cstr.ed.ac.uk/projects/festival

[19] C. Jayawardena, K. Watanabe, and K. Izumi, "Posture control of robot manipulators with fuzzy voice commands using a fuzzy coachplayer system," Advanced Robotics, vol. 21, pp. 293-328, 2007.

[20] K. Watanabe, C. Jayawardena, and K. Izumi, "Intelligent interface using natural voice and vision for supporting the acquisition of robot behaviors," in Proc. 5th IEEE Conference on Sensors, Oct. 2006, pp. 374-377.

[21] C. Jayawardena, K. Watanabe, and K. Izumi, "Controlling a robot manipulator with fuzzy voice commands using a probabilistic neural network," Neural Computing Applications, vol. 16, no. 2, pp. 155-166, 2007.

[22] — - "Probabilistic neural network based learning from fuzzy voice commands for controlling a robot," in Proceedings of the international conference on control, automation, and systems, Bangkok, Thailand, 2004.

[23] — - "Knowledge acquisition by a sub-coach in a coach player system for controlling a robot," in Proceedings of the 4th international conference on advanced mechatronics, Hokkaido, Japan, 2004, pp. 601-606. 